\title{
Src-mediated morphology transition of lung cancer cells in three-dimensional organotypic culture
}

\author{
Hong T Nguyen", Yan Zhuang ${ }^{1}$, Lichun Sun ${ }^{1}$, Steven P Kantrow², Jay K Kolls ${ }^{3}$, Zongbing You ${ }^{4}$, Ying Zhuo ${ }^{1}$ \\ and Bin Shan ${ }^{1 *}$
}

\begin{abstract}
A fribotic tumor microenvironment promotes progression of cancer. In this study, we utilize a reconstituted basement membrane mimics Matrigel based three-dimensional organotypic culture (rBM 3-D) to investigate the mechanisms that mediate the tumor promoting effects of the fibrogenic mediators TGF- $\beta 1$ and type I collagen (Col-1) on lung adenocarcinoma cells. Similar to normal alveolar epithelial cells, the well-differentiated lung adenocarcinoma cells in rBM 3-D culture undergo acinar morphogeneis that features polarized epithelial cell spheres with a single central lumen. Either TGF- $\beta 1$ or Col-1 modestly distorts acinar morphogenesis. On the other hand, TGF- $\beta 1$ and Col-1 synergistically induce a transition from acinar morphology into stellate morphology that is characteristic of invasive and metastatic cancer cells. Inhibition of the Src kinase activity abrogates induction of stellate morphology, activation of Akt and mTOR, and the expression of tumor promoting genes by TGF- $\beta 1$ and Col-1. To a similar extent, pharmacological inhibition of mTOR abrogates the cellular responses to TGF- $\beta 1$ and Col-1. In summary, we demonstrate that TGF- $\beta 1$ and Col-1 promote stellate morphogenesis of lung cancer cells. Our findings further suggest that the Src-Akt-mTOR axis mediates stellate morphogenesis. These findings also indicate that rBM 3-D culture can serve as an ideal platform for swift and cost-effective screening of therapeutic candidates at the interface of the tumor and its microenvironment.
\end{abstract}

Keywords: TGF- $\beta 1$, Src, Type I collagen, Three-dimensional culture, Extracellular matrix

\section{Background}

A stiff and fibrotic microenvironment promotes tumor progression in experimental models [1,2]. Accordingly, a fibrotic stroma is an independent prognostic indicator of metastasis and poor prognosis [3]. The majority of such evidence comes from the investigation of breast cancer in which the aberrantly stiff extracellular matrix (ECM) is a well-established risk factor [4]. A recent study has provided mechanistic insight into the link between the stiff ECM and progression of breast cancer [5]. Lysyl-oxidase (LOX) increases the stiffness of ECM via crosslinking collagen and thereby enhances integrin signaling to promote invasion and metastasis [5]. Recent advances in lung cancer research implicate a similar presence and function of a fibrotic tumor microenvironment. The expression of transforming growth factor- $\beta 1$ (TGF- $\beta 1)$ and type I

\footnotetext{
* Correspondence: bshan@tulane.edu

'Department of Medicine, Tulane University School of Medicine, 1430 Tulane Avenue, New Orleans, LA 70112, USA

Full list of author information is available at the end of the article
}

collagen (Col-1), two of the most potent fibrogenic mediators in the lung, is up-regulated in human lung cancer and overexpression of the two can promote invasion and metastasis in experimental models of lung cancer [6-8]. Elevated expression of LOX is a biomarker of invasion and an independent predictor of poor prognosis in patients with early stage lung adenocarcinoma [9]. In experimental models of lung cancer, LOX promotes tumor progression and is targeted by the tumor suppressor gene LKB1 [10]. However, the molecular mechanisms that mediate tumor progression promoted by the fibrotic tumor microenvironment in the lung remain poorly understood.

A substantial amount of our understanding of the tumor modulating functions of the tumor microenvironment has been obtained using three dimensional organotypic culture based on Matrigel, a reconstituted basement membrane mimics (rBM 3-D) [11-13]. rBM 3-D culture faithfully recapitulates salient in vivo properties of the epithelium from various tissues. The gene expression signature from rBM 3-D culture of breast cancer cells holds prognostic
C Biomed Central

(C) 2013 Nguyen et al.; licensee BioMed Central Ltd. This is an Open Access article distributed under the terms of the Creative Commons Attribution License (http://creativecommons.org/licenses/by/2.0), which permits unrestricted use, distribution, and reproduction in any medium, provided the original work is properly cited. 
value for breast cancer [14]. rBM 3-D culture is also a valuable tool to discriminate cancer cells with distinct tumorigenic potential [15]. In general, the non-invasive /metastatic breast cancer cells exhibit a mixture of acinar and mass morphology that features spheroid colonies (mass) with occasional formation of a single central lumen (acinus), whereas the invasive/metastatic cancer cells exhibit stellate morphology that features prominent invasive projections that often bridge multiple cell colonies. More importantly, rBM 3-D culture provides an ideal system to reconstitute the tumor microenvironment for mechanistic investigations. For instance, investigation of Col-1 and its cognate integrin receptors in rBM 3-D culture of mammary epithelial cells has identified the stiff ECM-integrin axis as a driving force of initiation and progression of breast cancer $[1,2,5]$. Two recent applications of rBM 3-D culture demonstrate its promise in elucidating molecular and cell biology of lung epithelial cells. In rBM 3-D culture, primary human lung alveolar type II cells form alveolar acini [16]. Similar to mammary epithelial cells, alveolar acini exhibit salient differentiation features, such as a polarized monolayer of alveolar type II cells and secretion of surfactant proteins into the central lumen. Because lung adenocarcinoma generally originates from alveolar type II cells, it is plausible that dysregulation of alveolar acini is a pivotal dedifferentiating step in lung tumorigenesis. In support of this concept, over-expression of the tumor suppressive PPAR- $\gamma$ gene can restore alveolar acini in rBM 3-D organotypic culture of H2122 cells, an aggressive and poorly differentiated human lung adenocarcinoma cell line [17].

Recent advances have shown that the tumor associated stroma and microenvironment are active modulators of tumorigenesis rather than passive bystanders [18]. The current study utilizes rBM 3-D organotypic culture to investigate a link between the behavior of lung cancer cells and the fribrogenic mediators derived from the tumor microenvironment.

\section{Results}

Morphogenesis of lung cancer cells in rBM 3-D culture rBM 3-D organotypic culture can promote differentiation of lung epithelial cells in vitro $[16,17]$. Therefore, we utilized this model to examine the effects of the fibrogenic mediators from the tumor microenvironment on morphogenesis of lung cancer cells. We established rBM 3-D culture of 4 human and mouse lung cancer cell lines with distinct tumorigenic properties. A549 cells are a welldifferentiated non-metastatic human lung adenocarcinoma cell line with residual characteristics of alveolar type II epithelial cells [19]. Similar to normal alveolar type II epithelial cells, A549 cells formed acini, a polarized cell sphere with a single central lumen in rBM 3-D culture (Figure 1A, inset) [16]. Moreover, acini formed by A549 cells in rBM 3-D culture resembled the glandular histology observed in the tumors formed by the implanted A549 cells in mice (Figure 1A). In contrast, A549LC cells, a more aggressive derivative of A549 cells (see Methods), exhibited mass morphology that featured irregular cell clusters void of a central lumen, which resembled the poorly differentiated H2122 cells in rBM 3-D culture as reported in a previous study (Figure 1B, inset) [17]. In congruence, the A549LC xenografts displayed disorganized structure and lacked the glandular histology (Figure 1B). Moreover, A549LC cells acquired greater tumorigenic activity than A549 cells in vivo because the implanted A549LC cells doubled the growth of the implanted parental A549 cells, $0.21 \pm 0.04$ g versus $0.1 \pm$ $0.03 \mathrm{~g}$ with marginal significance $(P$ value $=0.0678, \mathrm{n}=7$, Additional file 1: Figure S1). We further compared morphogenesis of two murine lung cancer cell lines $\mathrm{mK}$ ras-LE and LLC. mK-ras-LE cells were established from a tumor bearing lung of a K-ras ${ }^{L A 1}$ mouse, a transgenic strain that develops lung adenocarcinoma with limited metastasis [20]. Consistent with their well-differentiated phenotype, mK-ras-LE cells formed acini in rBM 3-D culture, which correlated with the glandular histology in the tumor formed by the implanted mK-ras-LE cells (Figure 1C). In contrast, the metastatic LLC cells exhibited stellate morphology that is characteristic of metastatic cancer cells (Figure 1D, inset) [15]. The stellate morphology featured irregular cell clusters with extensive intersecting cell protrusions (Figure 1D, inset). In accordance, the implanted LLC cells grew into irregular cell masses at the primary site and metastasized to the lung (Figure 1D and unpublished observations). The correlation of morphogenesis of four lung cancer cell lines in rBM 3-D culture and histology in vivo indicated that rBM 3-D culture is an appropriate in vitro model to assess morphogenesis that is relevant to tumorigenic behaviors of lung cancer cells in vivo.

\section{Src-mediated stellate morphogenesis induced by TGF- $\beta 1$ and Col-1}

Because the tumor microenvironment is commonly fibrotic and enriched with fibrogenic mediators, we examined morphogenesis of A549 and A549LC cells in rBM 3-D culture exposed to various combinations of TGF- $\beta 1$ and Col- 1 , two prominent fibrogenic mediators in the tumor microenvironment [6-8]. Addition of TGF- $\beta 1$ ( $5 \mathrm{ng} / \mathrm{ml})$ or Col-1 $(1.5 \mu \mathrm{g} / \mathrm{ml})$ alone caused little to modest perturbation of acini as evidenced by distorted cell clusters and partial to complete filling of central lumens (Figure 2A). Simultaneous exposure to TGF- $\beta 1$ and Col-1 abolished acinar morphology and induced a transition into stellate morphology that was characteristic of invasive/metastatic cancer cells (Figures 2A \& 1D) [15]. In a similar fashion, A549LC cells underwent transition from mass morphology into 

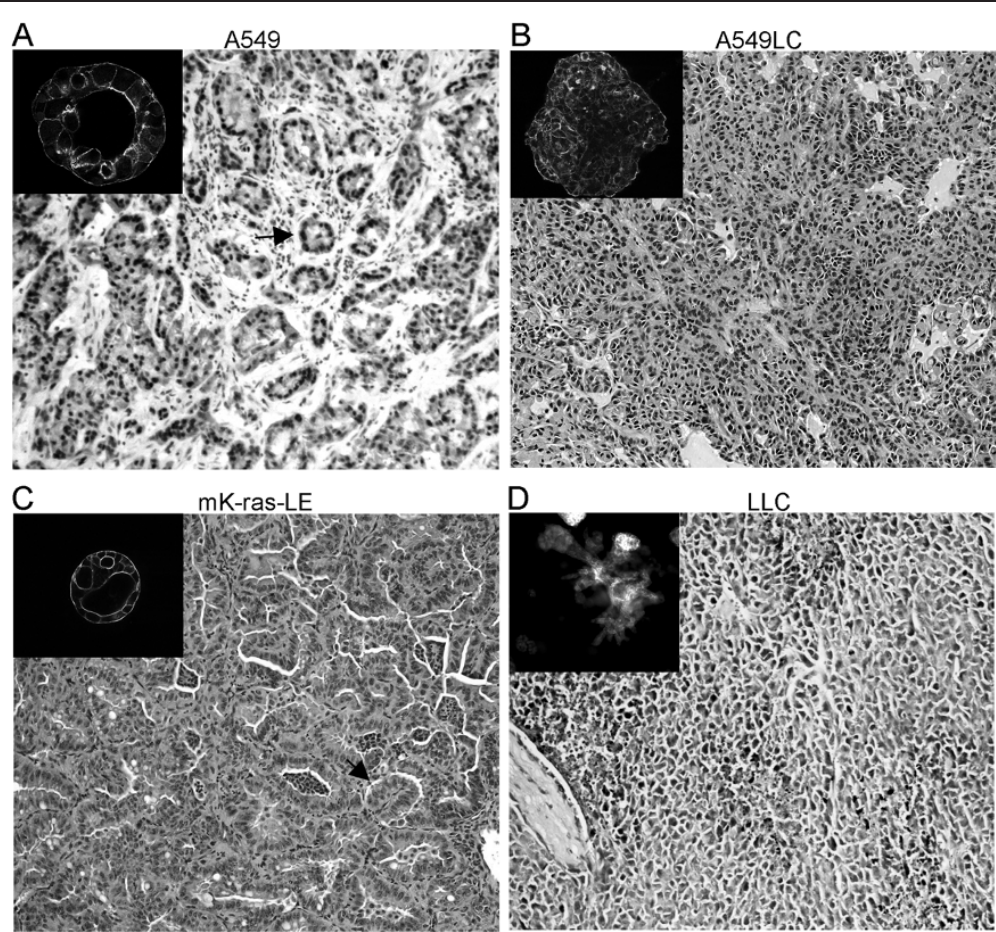

Figure 1 Distinct growth patterns of lung cancer cell lines in rBM 3-D organotypic culture. Four human and mouse lung cancer cell lines were cultured in rBM 3-D culture. Morphogenesis of lung cancer cells was visualized by filamentous actin staining and confocal fluorescent microscopy (insets). The insets of A-C were captured at 400x and part D was captured at 200x because of the larger diameter of LLC cell cluster. The growth patterns of lung cancer cells in vivo were visualized using $\mathrm{H}$ \& E staining (main images, 100x). Representative glandular structures are indicated by arrows in $\mathbf{A} \& \mathbf{C}$.

stellate morphology upon simultaneous exposure to TGF$\beta 1$ and Col-1 in rBM 3-D culture (Figure 2B).

The Src kinase is a key signal transducer of ECM and growth factors [21]. We then questioned whether the Src kinase activity is required for induction of stellate morphology by TGF- $\beta 1$ and Col- 1 . To this end, A549 cells were exposed to TGF- $\beta 1$ and Col-1 in the presence or absence of PP2 $(5 \mu \mathrm{M})$, an Src selective inhibitor. When compared to the group treated with the DMSO vehicle, PP2 abrogated induction of stellate morphology by TGF- $\beta 1$ and Col-1, but did not restore acinar morphology because the cell colonies were still void of a single central lumen (Figure 2C). Similar observations were made in A549LC cells upon exposure to various combinations of TGF- $\beta 1$, Col- 1 , and PP2 (data not shown). To further confirm a requirement of the Src kinase activity for induction of stellate morphology by TGF- $\beta 1$ and Col-1, we generated two variants of A549LC cells that were transduced with either a retroviral vector expressing a dominant-negative Src mutant (A549LCdnSrc) or its backbone vector (A549LCvec). Similar to PP2, the expression of the dnSrc mutant abolished stellate morphology induced by TGF- $\beta 1$ and Col-1, whereas A549LCvec's response to TGF- $\beta 1$ and Col- 1 was comparable to that of the parental A549LC cells (Figure 2, B \& D). These findings indicated a requirement of the Src kinase activity for induction of stellate morphology by TGF- $\beta 1$ and Col-1.

To elucidate the mechanisms underlying induction of stellate morphology, we examined the expression of three tumor-promoting genes, namely Myc, LOX, and plasminogen activator inhibitor-1 (PAI-1) because of their established link to TGF- $\beta 1$ and Col-1 $[5,6,8]$. The mRNA levels of these genes were determined using quantitative RT-PCR in A549 cells under various culture conditions. TGF- $\beta 1$ alone induced a robust increase in the expression of all three genes over the control group (3.6-fold in Myc, 303-fold in PAI-1, and 29.7-fold in LOX) (Figure 3, A-C). In contrast, Col-1 alone did not cause noticeable alteration in the expression of these genes. Despite the synergistic induction of stellate morphology, combination of TGF- $\beta 1$ and Col- 1 did not result in synergistic increase in the expression of these genes (Figure 3, A-C). These findings indicated that activation of the Myc, PAI- 1 , and LOX genes were by and large driven by the TGF- $\beta 1$ pathway during transition towards stellate morphology. Because inhibition of Src abolished stellate morphology induced by TGF- $\beta 1$ and Col- 1 , we examined the effects of PP2 on the induction of Myc, PAI- 1 , and LOX by TGF- $\beta 1$ and Col- 1 in rBM 3-D culture of A549LC cells. As expected, PP2 substantially reduced the induction of Myc (reduced to 


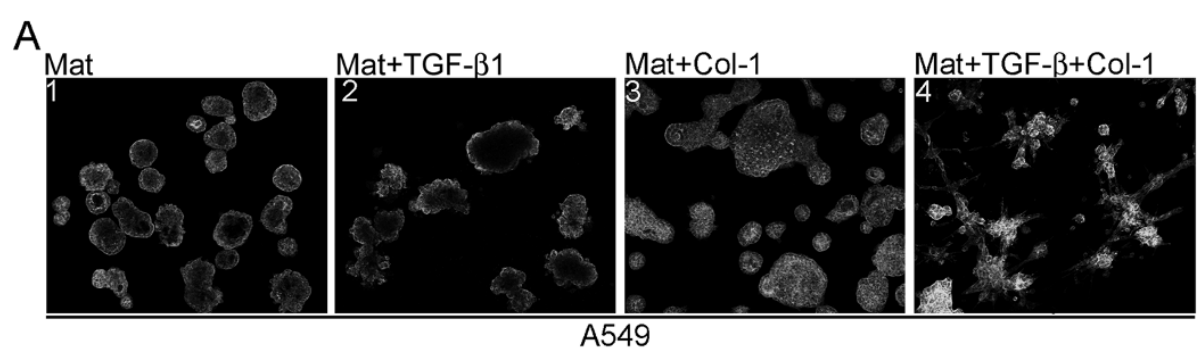

B
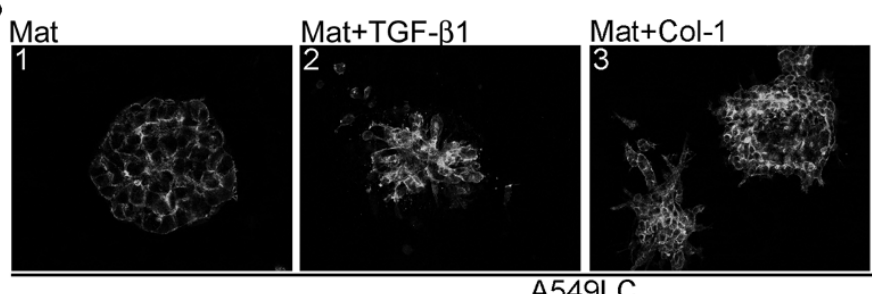

Mat+TGF- $\beta+\mathrm{Col}-1$

C

Mat Mat+Col-1+TGF- $\beta 1$ Mat+Col-1+TGF- $\beta 1+$ PP2


A549

$\mathrm{D}$

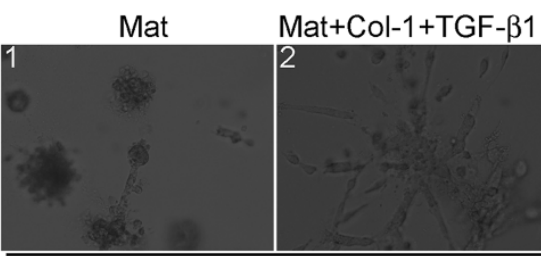

A549LCvec

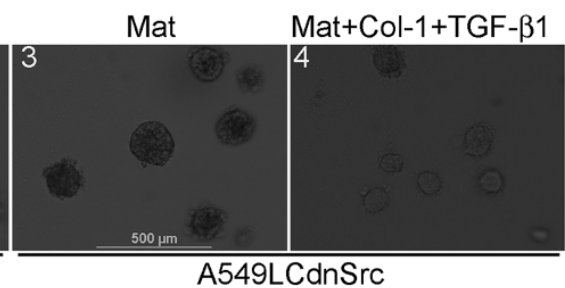

Figure 2 Induction of stellate morphology by TGF- $\beta 1$ and Col-1. A549 and its more aggressive derivative A549LC cells were cultured in rBM 3-D culture (Mat) with or without supplementation of TGF- $\beta 1$ and/or Col-1. A) Morphogenesis of A549 cells in various culture conditions were visualized by filamentous actin staining and confocal fluorescent microscopy. The images were captured at 100x. B) Similar to part A except that the images were captured from A549LC cells at 200x. C) Phase-contrast microscopy was used to capture images of A549 cells cultured in rBM 3D (Mat) exposed to the indicated combinations of TGF- $\beta 1$, Col-1, and PP2 (100x). D) The variants of A549LC cells, A549LCvec and A549LCdnSrC were cultured in rBM 3-D (Mat) in the present or absence of TGF- $\beta 1+C o l-1$ and morphogenesis was monitored as described in part C.

37\%), PAI-1 (reduced to 7\%), and LOX (reduced to 5\%) (Figure 3D). PP2 also inhibited TGF- $\beta 1$-induced expression of Myc (reduced to 24\%), PAI-1 (redcued to 6\%), and LOX (reduced to 8\%) (Figure 3E). Similar observations were made in A549LCvec and A549LCdnSrc cells (data not shown). These findings indicated a requirement of the Src kinase activity for induction of the Myc, PAI-1, and LOX genes by TGF- $\beta 1$ in rBM 3-D culture.

\section{Activation of the Akt-mTOR axis}

Src mediates activation of the Akt-mTOR axis in certain experimental conditions [22,23]. Because the Src kinase activity is required for stellate morphogenesis induced by TGF- $\beta 1$ and Col-1, we questioned whether the AktmTOR axis was activated by TGF- $\beta 1$ and Col- 1 in an Src-dependent manner. TGF- $\beta 1$ alone activated Src in
rBM 3-D culture because TGF- $\beta 1$ increased phosphorylation of Src at ser416 (Figure 4A). In contrast, Col-1 did not activate Src in rBM 3-D culture (Figure 4A). Moreover, a combination of TGF- $\beta 1$ and Col- 1 did not further increase the levels of Src phosphorylated at Ser416 (Figure 4A). In a similar fashion, the Akt-mTOR axis was activated by TGF- $\beta 1$ regardless of the presence of Col-1 because Akt became hyper-phosphorylated at Ser473 and mTOR became hyper-phosphorylated at Ser2448, a target site of Akt (Figure 4A). To determine whether the Src kinase activity was required for activation of the Akt-mTOR axis, we compared phosphorylation of Akt and mTOR in A549LCvec and A549LCdnSrc in rBM 3-D culture exposed to TGF- $\beta 1$ and Col-1 (Figure 4B). The dominant-negative activity of the dnSrc mutant was confirmed as A549LCdnSrc 


\section{A}

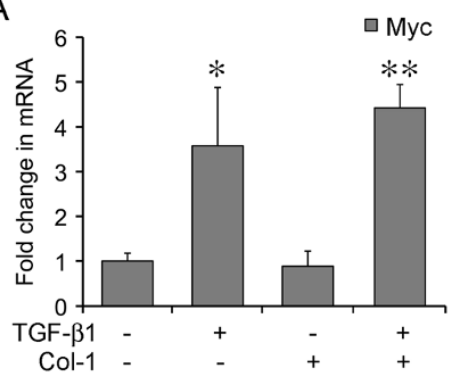

C



B

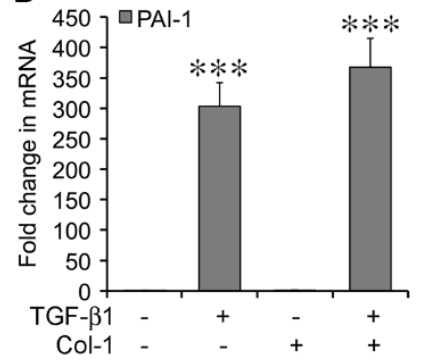

D



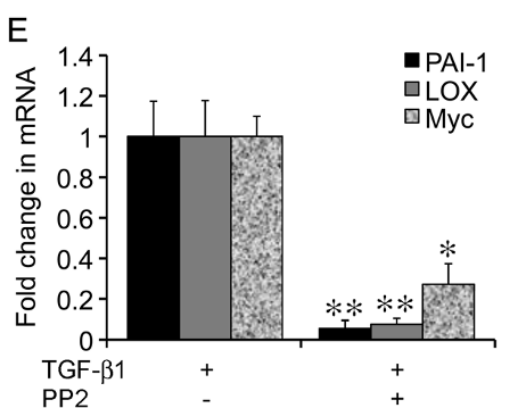

Figure 3 Activated expression of the tumor promoting genes by TGF- $\beta 1$ in rBM 3-D. Total cell RNA was extracted from A549 cells cultured in rBM 3-D (Mat) culture exposed to the indicated combinations of TGF- $\beta 1$ and Col-1. The expression of the selected tumor-promoting genes was determined using quantitative RT-PCR. Ratios of each mRNA over the housekeeping gene 36B4 were compared across various culture conditions. A-C) The expression of Myc, PAl-1, and LOX was evaluated, respectively. A fold change was obtained by setting the values from the control group to one. D) The expression of Myc, PAl-1, and LOX was compared between A549 cells cultured in Mat+TGF- $\beta 1+$ Col-1 with or without PP2. A fold change was obtained by setting the values from the PP2 minus group to one. E) Similar to part C except that the expression of Myc, PAl-1, and LOX was compared between A549 cells cultured in Mat+TGF- $\beta 1$ with or without PP2. Mean and standard deviations were obtained from three independent experiments. ${ }^{*}, * *$, and ${ }^{* * *}$ indicate a $P$ value $<0.05,0.01$, and 0.001 , respectively.

exhibited a reduced phosphorylation at Tyr861 in focal adhesion kinase (FAK), a classical target of Src (Figure 4B) [24]. As expected, A549LCdnSrc cells exhibited a substantial decrease in phosphorylation of Ser473 in Akt (Figure 4B). Consistent with reduced activation of Akt, A549LCdnSrc exhibited reduced phosphorylation of Ser2448 in mTOR (Figure 4B). Lastly, we examined phosphorylation of Thr389 in p70 S6K, a target site of mTOR. The expression of the dnSrc mutant substantially reduced phosphorylation of Thr389 in p70 S6K. These findings indicated a requirement of the Src kinase activity for activation of the Akt-mTOR axis by TGF- $\beta 1$ and Col-1. These results also prompted us to determine whether mTOR was required for induction of stellate morphology by TGF- $\beta 1$ and Col- 1 . To this end, A549 cells were cultured in rBM 3-D culture exposed to TGF- $\beta 1$ and Col-1 in the presence or absence of Torin-1 (250 nM), an mTOR-specific inhibitor [25]. As expected, Torin-1 abrogated stellate morphology induced by TGF- $\beta 1$ and Col- 1 (Figure 4B). Moreover, Torin-1 attenuated the gene activation by TGF- $\beta 1$ and Col-1 in that induction of the LOX and Myc genes was nearly abrogated, although induction of PAI-1 was refractory to Torin-1 (Figure 4C). These findings indicated that Src-mediated activation of the AktmTOR axis was required for stellate morphogenesis induced by TGF- $\beta 1$ and Col-1. 


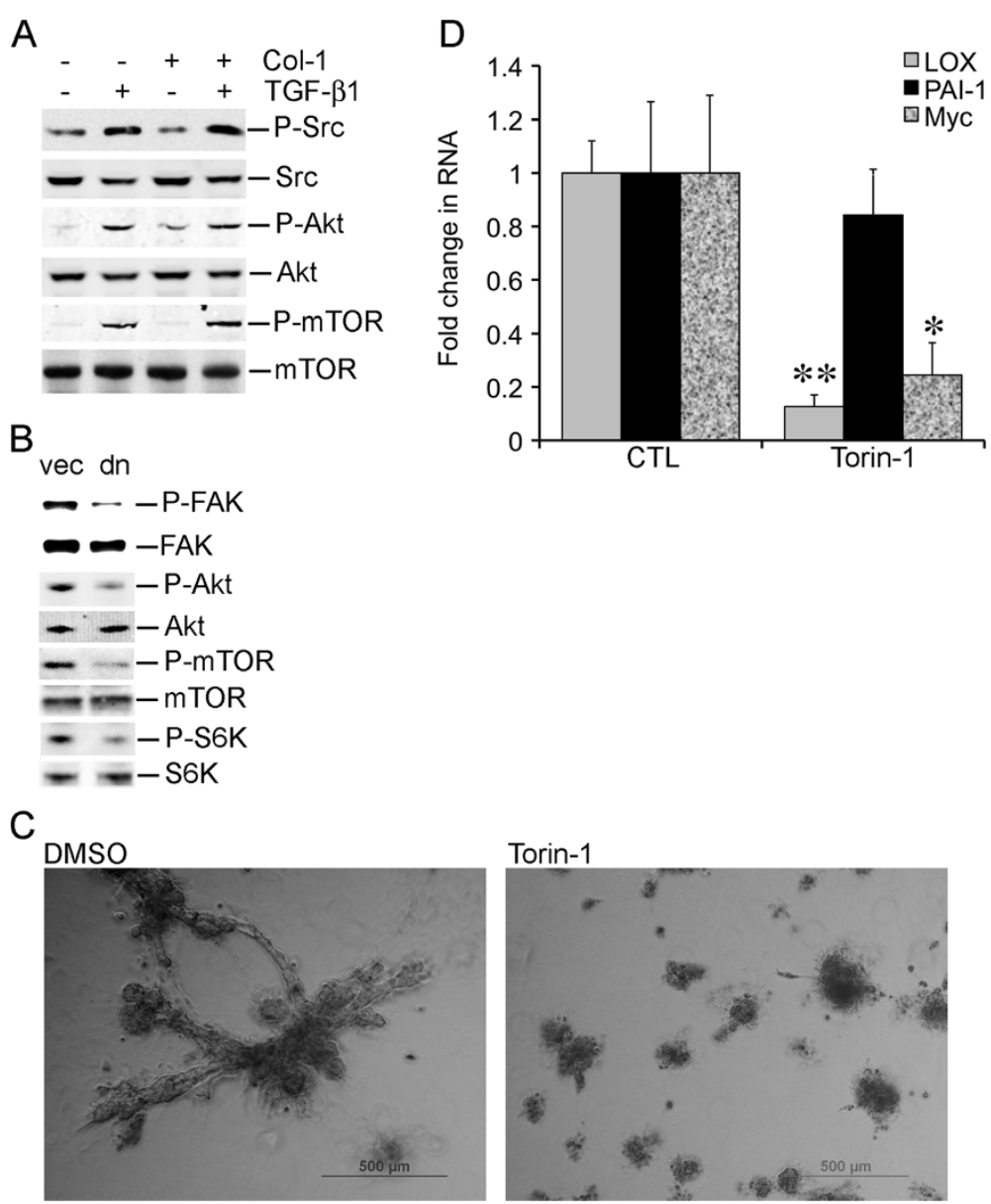

Figure 4 Activation of the Akt-mTOR axis in stellate morphogenesis. A) Total cell protein was extracted from A549 cells in rBM 3-D culture exposed to the indicated combinations of TGF- $\beta 1$ and Col-1. The protein levels of total and phosphorylated Src, Akt, and mTOR were determined using immunoblots. B) Similar to A except that total cell protein was extracted from A549LCvec and A549LCdnSrc cells in rBM 3-D culture exposed to TGF- $\beta 1$ and Col-1. The protein levels of total and phosphorylated FAK, Akt, mTOR, and p70 S6K were determined using immunoblots. C) A549LC cells were cultured in rBM 3D culture supplemented with TGF- $\beta 1$ and Col-1 in the presence or absence of Torin-1 (250 nM). Morphogenesis of A549LC cells was monitored for 12 days. Images were captured using phase contrast microscopy. D) The culture condition was identical to that described in part B. Total cell RNA was extracted from A549 cells. The expression of LOX, PAl-1, and Myc was determined using quantitative RT-PCR. Ratios of each gene transcript over the housekeeping gene $36 \mathrm{~B} 4$ were compared across various culture conditions. A fold change was obtained by setting the values from the control group to one. Mean and standard deviations were obtained from three independent experiments. ${ }^{*}$ and ${ }^{* *}$ indicate a $\mathrm{P}$ value $<0.05$ and 0.01 , respectively.

\section{Discussion}

The current study investigates the molecular mechanisms that mediate cancer progression promoted by the fibrotic tumor microenvironment using rBM 3-D culture of lung cancer cells. We aim to define the molecular mechanisms that mediate the tumor-promoting effects derived from the fibrotic tumor microenvironment.

rBM 3-D culture has been successfully applied to characterize molecular and cell biology of normal and transformed mammary epithelial cells for the past two decades [11-13]. In essence, rBM 3-D culture bears similar potential for investigation of lung biology because the lung and the breast share several key developmental and structural traits, such as branching morphogenesis during development and formation of alveoli [26]. Indeed, rBM 3-D culture of normal human lung alveolar type II epithelial cells promotes expression of the differentiation markers, such as surfactant protein $\mathrm{C}$ and formation of acini, an in vitro mimic of alveoli [16]. More importantly, over-expression of PPAR- $\gamma$, a tumor suppressor gene, can restore formation of acini in a poorly differentiated human lung cancer cell line in rBM 3-D culture [17]. Our findings strengthen the concept that rBM 3-D culture can be used to assess invasive and metastatic potential of lung cancer cells by comparing morphogenesis of four lung cancer cell lines with distinct tumorigenic behaviors in vivo. By and large, the well-differentiated lung adenocaricnoma cells, A549 and 
mK-ras-LE, form acini, whereas the more aggressive A549LC and LLC cells exhibit mass and stellate morphology (Figure 1). The diverse growth patterns of these four lung cancer cell lines in rBM 3-D culture are congruent to their disparate histology and tumorigenic potential in vivo (Figure 1). It is noteworthy that rBM 3-D culture reveals distinct morphogenesis between A549 (acinar morphology) and A549LC (mass morphology), whereas the two cell lines appear nearly identical in 2-D culture (data not shown). The morphological difference in rBM 3-D is also congruent to their distinct histology and tumorigenic activity in vivo (Figure 1, A \& B). With further optimization and validation, rBM 3-D organotypic culture can be utilized as a surrogate to qualitatively and quantitatively assess tumorigenic properties of lung cancer cells.

One major advantage of rBM 3-D culture is that it allows assessment of tumor modulating cues derived from the tumor microenvironment [11-13]. As revealed in our study, TGF- $\beta 1$ and Col-1 synergistically induce stellate morphology, a hallmark feature of invasive/metastatic cancer cells (Figures 1 \& 2) [15]. This combined exposure may recapitulate the fibrotic tumor microenvironment in vivo where lung cancer cells are simultaneously and constantly exposed to a variety of fibrogenic mediators [6-8]. Induction of stellate morphology by a combination of TGF- $\beta 1$ and Col- 1 is also consistent with a previous study in which provisional ECM, such as fibronectin and Col-1 potentiates epithelial-mesenchymal transition (EMT) of alveolar type II epithelial cells in response to TGF- $\beta 1$ in 2-D culture [27]. Thus, stellate morphology induced by TGF- $\beta 1$ and Col- 1 can be perceived as a phenomenon of EMT in rBM 3-D culture, which allows investigation of EMT of lung cancer cells, a pivotal step towards invasion/metastasis in the context of ECM. In support of our notion, characterization of EMT using rBM 3-D culture has been proposed as a routine protocol based on initial success of this approach [28].

Our attempt to pinpoint the mediators of the synergistic induction of stellate morphology by TGF- $\beta 1$ and Col-1 results in limited success. Nevertheless, we identify the signaling pathway and target genes activated by the TGF- $\beta 1$ arm, which is not sufficient, but required for transition from acinar to stellate morphology (Figures 2, $3,4)$. Specifically, the Src kinase activity is required for induction of stellate morphology and activation of gene expression by TGF- $\beta 1$ in the presence or absence of Col-1 (Figures 2 \& 3). Similarly, the Src kinase activity appears to be essential for activation of the Akt-mTOR axis by TGF- $\beta 1$ in the presence or absence of Col-1 (Figures 2 and 4). Besides the inducible stellate morphogenesis, the Src kinase activity appears to be required for native stellate morphogenesis of the invasive/metastatic cancer cell lines because inhibition of the Src kinase activity abrogates stellate morphogenesis of the invasive/ metastatic LLC, 4T1, and MDA-MB231 cells (unpublished observations).

Despite similar distortion of acinar morphogenesis, only TGF- $\beta 1$, but not Col-1 stimulates the expression of the MYC, LOX, and PAI-1 (Figures 1 \& 3). It is conceivable that Col-1 employs an alternative gene expression program to disrupt acinar morphogenesis. In support of this notion, Col-1 stimulates the expression of the oncogenic miR-21 gene in rBM 3-D culture, which is not observed in lung cancer cells exposed to TGF- $\beta 1$ (unpublished observations) [29]. Among the TGF- $\beta 1$-activated tumor promoting genes, LOX exhibit an Src- and mTORdependence and a strong correlation to stellate morphology (Figures 3 \& 4) [9]. These findings suggest a novel mechanism for the elevated expression of LOX in human lung cancer in that TGF- $\beta 1$ induces the expression of LOX in lung cancer cells via the Src-Akt-mTOR axis. It is also conceivable that the TGF- $\beta 1$-induced expression of LOX in rBM 3-D culture crosslinks the supplemented Col-1 to substantially increase the stiffness of rBM 3-D culture and thereby mediates synergistic induction of stellate morphology by TGF- $\beta 1$ and Col-1. Among the three genes examined upon blockade of Src and mTOR, PAI-1 appears to be refractory to inhibition of mTOR, whereas inhibition of Src diminishes activation of all three genes (Figures 3 \& 4). This suggests that mTOR mediate only part of the gene activation program activated by Src upon exposure to TGF- $\beta 1$. This observation could also be attributed to the SMAD3 binding motif in the PAI-1 promoter that induces the expression of PAI-1 through SMADs, the canonical TGF- $\beta$ pathway and delivers resistance to blockade of the non-canonical TGF- $\beta$ pathways, such as mTOR [30].

In summary, we demonstrate that the fibrogenic mediators derived from the tumor microenvironment promote stellate morphogenesis of lung cancer cells. Our results further suggest that the Src-Akt-mTOR axis, a group of promising therapeutic targets in lung cancer, acts as a signal transducer of the fibrotic tumor microenvironment $[22,23,31]$. Our work warrants further investigation to elucidate the molecular mechanisms that mediate synergistic induction of stellate morphology by TGF- $\beta 1$ and Col-1. These findings also strongly suggest that rBM 3-D culture can serve as an ideal platform for swift and costeffective screening of therapeutic candidates at the interface of the tumor and its microenvironment.

\section{Methods}

\section{Reagents and plasmids}

PP2, an Src specific inhibitor, was purchased from Calbiochem (San Diego, CA). Matrigel was purchased from BD Biosciences (Rockville, MD). Rat Col-1 was purchased from Sigma (St. Louis, MO). Recombinant 
human TGF- $\beta 1$ was obtained from R\&D Systems (Minneapolis, MN). A dominant-negative chicken Src-K295R mutant expressing retroviral vector $(\mathrm{dnSrc})$ and its backbone (pLNCX) were kindly provided by Dr. Brugge at Harvard University [32,33]. Torin1, an mTOR-specific inhibitor was a gift from Dr. Sabatini at MIT [25]. Invitrogen (Carlsbad CA) provided the antibodies specific for total and phosphorylated (Tyr861) FAK. Cell Signaling (Danvers MA) provided the antibodies specific for total and phosphorylated Src (Tyr416), Akt (Ser473), mTOR (Ser2448), and p70 S6K (Thr389).

\section{Cell culture}

A549 cells, a human lung adenocarcinoma cell line were obtained from ATCC (Manassas VA) and cultured as previously described [34,35]. A549LC cells were derived from parental A549 cells using a murine model of lung metastasis [36,37]. Briefly, A549 cells $\left(10^{6}\right.$ cells/mouse) were injected via the jugular vein into adult female beigeSCID mice (Charles River). Four months after injection, lungs were inspected and one metastatic nodule was excised, disaggregated and established in culture. The dnSrc expressing variant of A549LC (A549LCdnSrc) and its matching backbone vector variant (A549LCvec) were generated using retroviral transduction as we previously described [38]. mK-ras-LE cells, a murine lung epithelial cell line, were established from a tumor bearing lung of a K-ras ${ }^{L A 1}$ transgenic mouse and cultured in RPMI-1640 as described elsewhere [20,29]. Lewis lung carcinoma cells (LLC), a metastatic murine lung cancer cell line, were purchased from ATCC (Manassas, VA) and cultured in DMEM.

\section{rBM 3-D organotypic culture and image analysis}

rBM 3-D organotypic culture was employed because of the prior success of this approach in characterizing differentiation of both primary and transformed lung epithelial cells [11-13,16,17]. Briefly, the lung cancer cells were seeded in an overlay fashion on a layer of Matrigel on day zero. The culture medium containing 4\% Matrigel (volume/volume) was replaced every other day. Formation of acini was monitored for twelve days prior to harvest for image, RNA, and protein analyses. The cultured cells were visualized using fluorescent staining for filamentous actin with Alexa 488 conjugated phalloidin (Invitrogen, Carlsbad $\mathrm{CA}$ ). The images were captured using confocal fluorescent or phase contrast microscopy as we previously described $[26,39]$. In the selected cultures, various combinations of TGF- $\beta 1$ (5 ng/ml), Col-1 $(1.5 \mu \mathrm{g} / \mathrm{ml})$, and Torin-1 (250 nM) were added to rBM 3-D culture.

\section{RNA extraction and quantitative RT-PCR}

Total cell RNA was extracted from rBM 3-D culture using TRIzol per the provider's instructions (Invitrogen, Carlbad
CA). The expression of each gene of interest was determined using quantitative RT-PCR on an iCycler (BIORAD, Hercules CA) and compared across the groups as described else where [40]. The sequences of each pair of primers were listed in Additional file 1: Table S1.

\section{Immunoblots}

Total cell protein was extracted using RIPA buffer supplemented with Protease and Protein Phosphatase Inhibitor Cocktails (Sigma, St. Louis MO) after A549 cells and their variants were extracted from rBM 3-D culture [26]. The expression of the total and phosphorylated proteins of interest was determined using immunoblots as described we previously described [34].

\section{Implantation of lung cancer cells}

All mouse studies were carried out following the animal protocol approved by the Institute Animal Care and Use Committee at Tulane University School of Medicine. Subcutaneous implantation of human and mouse lung cancer cells $\left(2 \times 10^{6}\right.$ cells/mouse $)$ into male nude and syngeneic mice (Charles River, Wilmington, MA) was carried out as we previously described $[36,41]$. Each group of tumor graft consisted of 7 mice. Tumor growth was monitored daily after implantation. The tumor mass was dissected from mice at four weeks after implantation and processed for weighing and $H \& E$ staining.

\section{Statistical analysis}

When presented, means and standard deviations were obtained from 3 independent experiments. A $P$ value between any two selected groups was determined using unpaired two-tailed Student's T-test (GraphPad Prism, Version 5).

\section{Additional file}

Additional file 1: Table S1. Primers. Figure S1 Tumor growth of subcutaneously implanted A549 and A549LC cells.

\section{Abbreviations}

ECM: Extracellular matrix; LOX: Lysyl-oxidase; TGF- $\beta 1$ : Transforming growth factor- $\beta 1$; Col-1: Type I collagen; mTOR: Mammalian target of rapamycin; PAI1: Plasminogen activator inhibitor-1.

\section{Competing interests}

The authors declare no competing interests.

\section{Authors' contribution}

HTN carried out rBM 3-D culture, image analysis, qRT-PCR, and immunoblots. YZ carried out qRT-PCR experiments and immunoblots. LS and YZ carried out xenograft studies of A549 and mK-ras-LE cells. SPK and JKK generated A549LC cells and carried out xenograft studies of A549LC cells. ZY carried out xenograft studies of LLC cells. BS conceived the study and wrote the manuscript. All authors read and approved the final manuscript. 


\section{Acknowledgements}

This work is supported by Tulane Research Enhancement Fund. We are grateful to Dr. Brugge and Dr. Sabatini for providing the Src mutant and the mTOR inibitor, respectively.

\section{Author details}

${ }^{1}$ Department of Medicine, Tulane University School of Medicine, 1430 Tulane Avenue, New Orleans, LA 70112, USA. ${ }^{2}$ Department of Medicine, Louisiana State University Health Sciences Center, 1901 Perdido Street, New Orleans, LA 70112, USA. ${ }^{3}$ Department of Pediatrics, University of Pittsburgh School of Medicine, Pittsburgh, PA 15224, USA. ${ }^{4}$ Department of Structural and Cellular Biology, Tulane University School of Medicine, 1430 Tulane Avenue, New Orleans, LA 70112, USA.

Received: 3 October 2012 Accepted: 11 February 2013 Published: 14 February 2013

\section{References}

1. Paszek MJ, Zahir N, Johnson KR, Lakins JN, Rozenberg GI, Gefen A, ReinhartKing CA, Margulies SS, Dembo M, Boettiger D, et al: Tensional homeostasis and the malignant phenotype. Cancer Cell 2005, 8(3):241-254.

2. Bian YF, Yang HY, Yang ZM, Gao F, Zhang NN, Xiao CS: Amlodipine treatment prevents angiotensin II-induced human umbilical vein endothelial cell apoptosis. Arch Med Res 2011, 42(1):22-27.

3. Kalluri R, Zeisberg M: Fibroblasts in cancer. Nat Rev Cancer 2006, 6(5):392-401.

4. Provenzano PP, Eliceiri KW, Campbell JM, Inman DR, White JG, Keely PJ: Collagen reorganization at the tumor-stromal interface facilitates local invasion. BMC Med 2006, 4(1):38.

5. Levental KR, Yu H, Kass L, Lakins JN, Egeblad M, Erler JT, Fong SF, Csiszar K, Giaccia A, Weninger W, et al: Matrix crosslinking forces tumor progression by enhancing integrin signaling. Cell 2009, 139(5):891-906.

6. Borczuk AC, Papanikolaou N, Toonkel RL, Sole M, Gorenstein LA, Ginsburg $M E$, Sonett JR, Friedman RA, Powell CA: Lung adenocarcinoma invasion in TGFbetaRIl-deficient cells is mediated by CCL5/RANTES. Oncogene 2008, 27(4):557-564

7. Han S, Khuri FR, Roman J: Fibronectin stimulates non-small cell lung carcinoma cell growth through activation of Akt/mammalian target of rapamycin/S6 kinase and inactivation of LKB1/AMP-activated protein kinase signal pathways. Cancer Res 2006, 66(1):315-323.

8. Soltermann A, Tischler V, Arbogast S, Braun J, Probst-Hensch N, Weder W, Moch H, Kristiansen G: Prognostic significance of epithelial-mesenchymal and mesenchymal-epithelial transition protein expression in non-small cell lung cancer. Clin Cancer Res 2008, 14(22):7430-7437.

9. Borczuk AC, Sole M, Lu P, Chen J, Wilgus ML, Friedman RA, Albelda SM, Powell CA: Progression of human bronchioloalveolar carcinoma to invasive adenocarcinoma is modeled in a transgenic mouse model of K-ras-induced lung cancer by loss of the TGF-beta type II receptor. Cancer Res 2011, 71(21):6665-6675.

10. Gao Y, Xiao Q, Ma H, Li L, Liu J, Feng Y, Fang Z, Wu J, Han X, Zhang J, et al: LKB1 inhibits lung cancer progression through lysyl oxidase and extracellular matrix remodeling. Proc Natl Acad Sci USA 2010, 107(44):18892-18897.

11. Debnath J, Muthuswamy SK, Brugge JS: Morphogenesis and oncogenesis of MCF-10A mammary epithelial acini grown in three-dimensional basement membrane cultures. Methods 2003, 30(3):256-268.

12. Hebner C, Weaver VM, Debnath J: Modeling morphogenesis and oncogenesis in three-dimensional breast epithelial cultures. Annu Rev Pathol 2008, 3:313-339

13. Lee GY, Kenny PA, Lee EH, Bissell MJ: Three-dimensional culture models of normal and malignant breast epithelial cells. Nat Methods 2007, 4(4):359-365.

14. Martin KJ, Patrick DR, Bissell MJ, Fournier MV: Prognostic breast cancer signature identified from 3D culture model accurately predicts clinical outcome across independent datasets. PLoS One 2008, 3(8):e2994.

15. Kenny PA, Lee GY, Myers CA, Neve RM, Semeiks JR, Spellman PT, Lorenz K, Lee EH, Barcellos-Hoff MH, Petersen OW, et al: The morphologies of breast cancer cell lines in three-dimensional assays correlate with their profiles of gene expression. Mol Oncol 2007, 1(1):84-96.

16. Yu W, Fang X, Ewald A, Wong K, Hunt CA, Werb Z, Matthay MA, Mostov K: Formation of cysts by alveolar type II cells in three-dimensional culture reveals a novel mechanism for epithelial morphogenesis. Mol Biol Cell 2007, 18(5):1693-1700.
17. Bren-Mattison $Y$, Van Putten $V$, Chan D, Winn R, Geraci MW, Nemenoff RA: Peroxisome proliferator-activated receptor-gamma (PPAR(gamma)) inhibits tumorigenesis by reversing the undifferentiated phenotype of metastatic non-small-cell lung cancer cells (NSCLC). Oncogene 2005, 24(8):1412-1422.

18. De Palma M, Hanahan D: The biology of personalized cancer medicine: Facing individual complexities underlying hallmark capabilities. Mol Oncol 2012, 6(2):111-127

19. Wang $D$, Haviland DL, Burns AR, Zsigmond E, Wetsel RA: A pure population of lung alveolar epithelial type II cells derived from human embryonic stem cells. Proc Natl Acad Sci USA 2007, 104(11):4449-4454.

20. Johnson L, Mercer K, Greenbaum D, Bronson RT, Crowley D, Tuveson DA Jacks T: Somatic activation of the K-ras oncogene causes early onset lung cancer in mice. Nature 2001, 410(6832):1111-1116.

21. Playford MP, Schaller MD: The interplay between Src and integrins in normal and tumor biology. Oncogene 2004, 23(48):7928-7946.

22. Lee MY, Lee SH, Park JH, Han HJ: Interaction of galectin-1 with caveolae induces mouse embryonic stem cell proliferation through the Src, ERas, Akt and mTOR signaling pathways. Cell Mol Life Sci 2009, 66(8):1467-1478.

23. Anand S, Muthusamy VS, Sujatha S, Sangeetha KN, Bharathi Raja R, Sudhagar S, Poornima Devi N, Lakshmi BS: Aloe emodin glycosides stimulates glucose transport and glycogen storage through PI3K dependent mechanism in L6 myotubes and inhibits adipocyte differentiation in 3T3L1 adipocytes. FEBS Lett 2010, 584(14):3170-3178.

24. Calalb MB, Zhang X, Polte TR, Hanks SK: Focal adhesion kinase tyrosine861 is a major site of phosphorylation by Src. Biochem Biophys Res Commun 1996, 228(3):662-668.

25. Thoreen CC, Kang SA, Chang JW, Liu Q, Zhang J, Gao Y, Reichling LJ, Sim T, Sabatini DM, Gray NS: An ATP-competitive mammalian target of rapamycin inhibitor reveals rapamycin-resistant functions of mTORC1. J Biol Chem 2009, 284(12):8023-8032.

26. Affolter $\mathrm{M}$, Zeller $\mathrm{R}$, Caussinus $\mathrm{E}$ : Tissue remodelling through branching morphogenesis. Nat Rev Mol Cell Biol 2009, 10(12):831-842.

27. Kim KK, Kugler MC, Wolters PJ, Robillard L, Galvez MG, Brumwell AN, Sheppard D, Chapman HA: Alveolar epithelial cell mesenchymal transition develops in vivo during pulmonary fibrosis and is regulated by the extracellular matrix. Proc Natl Acad Sci USA 2006, 103(35):13180-13185.

28. Moreno-Bueno G, Peinado H, Molina P, Olmeda D, Cubillo E, Santos V, Palacios J, Portillo F, Cano A: The morphological and molecular features of the epithelial-to-mesenchymal transition. Nat Protoc 2009, 4(11):1591-1613,

29. Li C, Nguyen HT, Zhuang Y, Lin Y, Flemington EK, Guo W, Guenther J, Burow ME, Morris GF, Sullivan D, et al: Post-transcriptional up-regulation of miR-21 by type I collagen. Mol Carcinog 2011, 50(7):563-570

30. Wrana JL, Attisano L, Carcamo J, Zentella A, Doody J, Laiho M, Wang XF, Massague J: TGF beta signals through a heteromeric protein kinase receptor complex. Cell 1992, 71(6):1003-1014.

31. Dy GK, Adjei AA: Emerging therapeutic targets in non-small cell lung cancer. Proc Am Thorac Soc 2009, 6(2):218-223.

32. Nagashima T, Maruyama T, Uchida H, Kajitani T, Arase T, Ono M, Oda H, Kagami M, Masuda H, Nishikawa S, et al: Activation of SRC kinase and phosphorylation of signal transducer and activator of transcription- 5 are required for decidual transformation of human endometrial stromal cells. Endocrinology 2008, 149(3):1227-1234.

33. Thomas JE, Soriano P, Brugge JS: Phosphorylation of c-Src on tyrosine 527 by another protein tyrosine kinase. Science 1991, 254(5031):568-571.

34. Shan B, Zhuo Y, Chin D, Morris CA, Morris GF, Lasky JA: Cyclin-dependent kinase 9 is required for tumor necrosis factor-alpha-stimulated matrix metalloproteinase-9 expression in human lung adenocarcinoma cells. J Biol Chem 2005, 280(2):1103-1111.

35. Zhuang Y, Nguyen HT, Lasky JA, Cao S, Li C, Hu J, Guo X, Burow ME, Shan B: Requirement of a novel splicing variant of human histone deacetylase 6 for TGF-beta1-mediated gene activation. Biochem Biophys Res Commun 2010, 392(4):608-613.

36. Bao L, Jaligam V, Zhang XY, Kutner RH, Kantrow SP, Reiser J: Stable transgene expression in tumors and metastases after transduction with lentiviral vectors based on human immunodeficiency virus type 1 . Hum Gene Ther 2004, 15(5):445-456.

37. Li C, Nguyen HT, Zhuang Y, Lin Z, Flemington EK, Zhuo Y, Kantrow SP, Morris GF, Sullivan DE, Shan B: Comparative profiling of miRNA expression of lung adenocarcinoma cells in two-dimensional and three-dimensional cultures. Gene 2012, 511(2):143-150. 
38. Shan B, Morris CA, Zhuo Y, Shelby BD, Levy DR, Lasky JA: Activation of proMMP-2 and Src by HHV8 VGPCR in human pulmonary arterial endothelial cells. J Mol Cell Cardiol 2007, 42(3):517-525.

39. Nguyen HT, Li C, Lin Z, Zhuang Y, Flemington EK, Burow ME, Lin Y, Shan B: The microRNA expression associated with morphogenesis of breast cancer cells in three-dimensional organotypic culture. Oncol Rep 2012 28(1):117-126.

40. Shan B, Hagood JS, Zhuo Y, Nguyen HT, MacEwen M, Morris GF, Lasky JA: Thy- 1 attenuates TNF-alpha-activated gene expression in mouse embryonic fibroblasts via Src family kinase. PLoS One 2010, 5(7):e11662.

41. Sun L, Vasilevich NI, Fuselier JA, Coy DH: Abilities of 3,4-diarylfuran-2-one analogs of combretastatin A-4 to inhibit both proliferation of tumor cell lines and growth of relevant tumors in nude mice. Anticancer Res 2004, 24(1):179-186

doi:10.1186/1475-2867-13-16

Cite this article as: Nguyen et al.: Src-mediated morphology transition of lung cancer cells in three-dimensional organotypic culture. Cancer Cell International 2013 13:16.

\section{Submit your next manuscript to BioMed Central and take full advantage of:}

- Convenient online submission

- Thorough peer review

- No space constraints or color figure charges

- Immediate publication on acceptance

- Inclusion in PubMed, CAS, Scopus and Google Scholar

- Research which is freely available for redistribution 